\begin{tabular}{|c|c|}
\hline Title & A $n$ effect of the Gilbert damping constant on the skyrmion Hall effect \\
\hline Author(s) & Ishida, Y uichi; Kondo, Kenji \\
\hline Citation & $\begin{array}{l}\text { Journal of Magnetism and Magnetic Materials, 493, } 165687 \\
\text { https://doi.org/10.1016/.jmmm.2019.165687 }\end{array}$ \\
\hline Issue Date & 2020-01-01 \\
\hline Doc URL & http:/hdl.handle.net/2115/83755 \\
\hline Rights & $\begin{array}{l}\text { (9 2020, Elsevier. Licensed under the Creative Commons A ttribution-NonCommercial-NoDerivatives } 4.0 \text { International } \\
\text { https://creativecommons.org/icenses/by-nc-nd/4.0/ }\end{array}$ \\
\hline Rights(URL) & https://creativecommons.org/icenses/by-nc-nd/4.0/ \\
\hline Type & article (author version) \\
\hline File Information & main_revised.pdf \\
\hline
\end{tabular}

Instructions for use 


\title{
An Effect of the Gilbert Damping Constant on the Skyrmion Hall effect
}

\author{
Yuichi Ishida, Kenji Kondo* \\ Research Institute for Electronic Science, Hokkaido University, Kita-20, Nishi-10, Sapporo, Hokkaido, Japan
}

\begin{abstract}
We investigate the skyrmion Hall effect using the Landau-Lifshitz-Gilbert (LLG) equation and the Thiele equation, respectively. Then, we find that these methods give different values for the ratio of in-plane skyrmion velocity components when the Gilbert damping constant is relatively small. Since the Thiele equation is derived from the LLG equation by assuming that the skyrmion structure does not change and behaves like a rigid body, the above result suggests that this assumption does not hold when the Gilbert damping constant is relatively small. Therefore, we conclude that the Thiele equation can not describe the systems precisely under the relatively small Gilbert damping constant due to the distortion of the skyrmion structure and that it is mandatory to solve the LLG equation numerically in order to investigate the skyrmion Hall effect accurately. This result is very important since the Gilbert damping constants of metal materials are generally very small.
\end{abstract}

Keywords: Skyrmion Hall effect, Micromagnetic simulation, LLG equation PACS: M 75.60.Ch, 75.70.Cn, N 75.78.-n, N 75.78.Cd, N 75.78.Fg

\section{Introduction}

Magnetic skyrmions are chiral spin structures which has particle-like properties [1]. Recently, skyrmions are considered to be candidates for spintronics devices such as high-density memories and logic devices. This is because skyrmions are small and can be driven by a low current density in comparison with the critical current density for domain wall motion [2]. Moreover, it is difficult to destroy their structures and deform them to other magnetic structures since their structures are topologically protected. This is another advantage to utilizing skyrmions for spintronics devices.

In heterostructures of insulator/ultrathin ferromagnet/ heavy metal multilayers, magnetic skyrmions can be moved by the spin orbit torque induced from a pure spin current which is generated by the spin Hall effect [3, 4]. The spin Hall effect occurs in the heavy metal layer when we apply the charge current along the $x$-direction as shown in Fig. 1. Then, skyrmions move perpendicularly to the above current flow, which is called the skyrmion Hall effect [5]. The skyrmion Hall effect is considered to be caused by the effective Magnus force acting on the skyrmion. A skyrmion has a topological invariant which is called skyrmion charge $Q$. The skyrmion charge $Q$ has integer number and skyrmions deflect to the right or the left direction depending on the sign of the skyrmion charge.

The skyrmion Hall effect can be investigated by the Thiele equation. However, this equation is derived as-

\footnotetext{
* Corresponding author

Email address: kkondo@es.hokudai.ac.jp (Kenji Kondo)
}

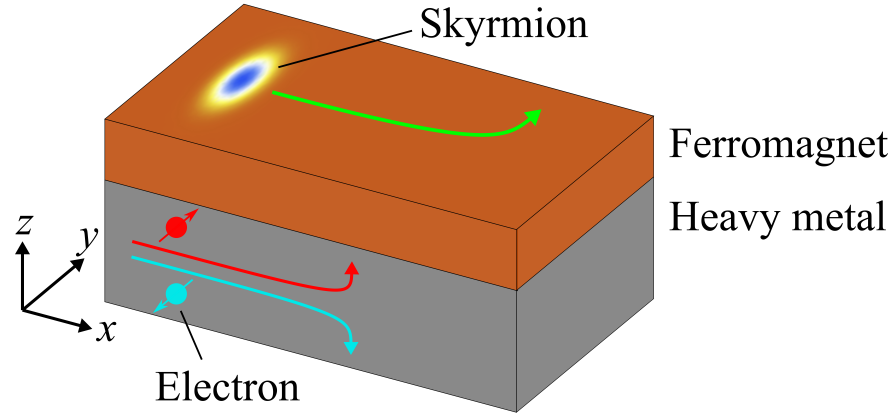

Figure 1: An illustration of the skyrmion Hall effect. A skyrmion in the ferromagnet layer is deflected by the spin orbit torque from the spin current generated in the heavy metal layer.

suming that the skyrmion structure does not change and behaves like a rigid body. Therefore, when the above assumption does not hold, it is considered that the Thiele equation becomes invalid.

In this study, we investigate the skyrmion Hall effect using both micromagnetic simulations and the Thiele equation and compare the results obtained by the two different methods. Moreover, the time evolution of the skyrmion with skyrmion charge of \pm 1 is demonstrated in detail.

\section{Theory}

The magnetization of an isolated skyrmion can be described by the spherical coordinates as follows:

$$
\boldsymbol{m}=(\cos \Phi(\phi) \sin \theta(r), \sin \Phi(\phi) \sin \theta(r), \cos \theta(r)),
$$


where $\boldsymbol{m}$ is the magnetization unit vector and $\Phi(\phi)=n \phi+$ $\gamma$. Here, $\phi$ is the azimuth angle, $n$ is the vorticity, and $\gamma$ is the helicity. We consider the case of $n=1$ and $\gamma=0$ or $\pi$ in this study. A skyrmion structure is obtained by calculating the lowest energy state of the magnetization given by Eq. (1) by utilizing the variational principle [6-9]. In our study, the total energy consists the exchange energy, the uniaxial perpendicular anisotropy energy, the interfacial Dzyaloshinskii-Moriya interaction (DMI) energy, and the demagnetization energy. As a result, the total energy density is given by

$$
\begin{aligned}
\varepsilon= & A\left[\left(\frac{\partial \boldsymbol{m}}{\partial x}\right)^{2}+\left(\frac{\partial \boldsymbol{m}}{\partial y}\right)^{2}+\left(\frac{\partial \boldsymbol{m}}{\partial z}\right)^{2}\right]+K\left(1-m_{z}^{2}\right) \\
& +D_{\mathrm{DMI}}\left[m_{z}\left(\frac{\partial m_{x}}{\partial x}+\frac{\partial m_{y}}{\partial y}\right)-\left(m_{x} \frac{\partial m_{z}}{\partial x}+m_{y} \frac{\partial m_{z}}{\partial y}\right)\right] \\
& +\frac{1}{2} \mu_{0} M_{s} \boldsymbol{m} \cdot \boldsymbol{H}_{\mathrm{d}}
\end{aligned}
$$

where $A$ is the exchange stiffness, $K$ is the perpendicular anisotropy constant, $D_{\mathrm{DMI}}$ is the DMI constant, $\mu_{0}$ is the vacuum permeability, $M_{s}$ is the saturation magnetization, and $\boldsymbol{H}_{\mathrm{d}}$ is the demagnetization field. For the demagnetization energy, we utilize the local approximation that the demagnetization field $\boldsymbol{H}_{\mathrm{d}}$ is determined by $\boldsymbol{H}_{\mathrm{d}}=-M_{s} m_{z} \hat{z}$. When we calculate the skyrmion structure, it is imposed that the boundary condition $\theta(0)=\pi$ and $\theta(\infty)=0$, or $\theta(0)=0$ and $\theta(\infty)=\pi$. Under this boundary condition, we obtain $\Phi(\phi)=\phi$ or $\Phi(\phi)=\phi+\pi$ to minimize the total energy, respectively. Then, we obtain the Euler-Lagrange equation of the angle of $\theta$ which is as follows:

$$
\frac{d^{2} \theta}{d \tilde{r}^{2}}+\frac{1}{\tilde{r}} \frac{d \theta}{d \tilde{r}}-\frac{\sin \theta \cos \theta}{\tilde{r}} \pm \frac{D_{\mathrm{DMI}}}{\sqrt{A K}} \frac{\sin ^{2} \theta}{\tilde{r}}-\sin \theta \cos \theta=0
$$

where $\tilde{r}=r \sqrt{K / A}$. The plus or minus sign of the fourth term corresponds to the boundary condition of $\theta(0)=$ $\pi$ and $\theta(\infty)=0$, or $\theta(0)=\pi$ and $\theta(\infty)=0$, respectively. The skyrmion structure obtained from Eq. (3) is distinguished by the topological invariant called skyrmion charge which is defined by

$$
Q=\frac{1}{4 \pi} \int \boldsymbol{m} \cdot\left(\frac{\partial \boldsymbol{m}}{\partial x} \times \frac{\partial \boldsymbol{m}}{\partial x}\right) d x d y .
$$

This corresponds to how many times the magnetization wraps the $S^{2}$ sphere. Inserting the Eq. (1) to the above, the skyrmion charge is explicitly given by

$$
Q=\frac{n}{2}\left(m_{z}(0)-m_{z}(\infty)\right)
$$

Therefore, the skyrmion charge becomes $Q= \pm n= \pm 1$ under the above conditions.

When the spin torque works on magnetization, the magnetization dynamics is governed by the modified LandauLifshitz-Gilbert (LLG) equation which is written by $[10$, $11]$

$$
\frac{d \boldsymbol{m}}{d t}=-\gamma \mu_{0} \boldsymbol{m} \times \boldsymbol{H}_{\mathrm{eff}}+\alpha \boldsymbol{m} \times \frac{d \boldsymbol{m}}{d t}+\frac{\boldsymbol{\tau}}{M_{s}},
$$

where $\gamma$ is the gyromagnetic ratio, $\alpha$ is the Gilbert damping constant, $\boldsymbol{H}_{\text {eff }}$ is the effective magnetic field, and $\boldsymbol{\tau}$ is the spin torque working on the magnetization. The effective magnetic field represents all the effects working on magnetic moments and can be obtained using functional derivative as follows:

$$
\boldsymbol{H}_{\mathrm{eff}}=-\frac{1}{\mu_{0}} \frac{\delta E[\boldsymbol{M}]}{\delta \boldsymbol{M}}
$$

where $E$ is the total energy of all the effects working on magnetic moments and $\boldsymbol{M}$ is the magnetization vector. When we calculate the effective magnetic field numerically, we employ a finite element method (FEM) for the space discretization. Specifically, after transforming the Eq. (7) to the next equation, we utilize the Rayleigh-Ritz method to solve the equation.

$$
\frac{\delta}{\delta \boldsymbol{M}}\left(E[\boldsymbol{M}]+\mu_{0} \boldsymbol{M} \cdot \boldsymbol{H}_{\mathrm{eff}}\right)=0 .
$$

When the effective magnetic field is calculated by this method, it is not necessary to take into consideration the following boundary condition explicitly [12].

$$
\frac{d \boldsymbol{m}}{d n}=\frac{D_{\mathrm{DMI}}}{2 A}(\hat{z} \times \boldsymbol{n}) \times \boldsymbol{m},
$$

where $\boldsymbol{n}$ is the unit vector perpendicular to the edge. This is because the above condition is included naturally within the process of solving the Eq. (8). Therefore, the RayleighRitz method has the benefit that all elements can be equally treated without dealing with the edges exceptionally. When the spin torque is generated from the spin Hall effect, the last term of Eq. (6) is written as follows [13-16]:

$$
\boldsymbol{\tau}=-\frac{\gamma \hbar}{2 e} \frac{\theta_{\mathrm{sh}}}{t_{f}} \boldsymbol{m} \times[\boldsymbol{m} \times(\hat{z} \times \boldsymbol{j})],
$$

where $\hbar$ is the Dirac constant, $e$ is the electron charge, $\theta_{\text {sh }}$ is the spin Hall angle of the heavy metal, $t_{f}$ is the thickness of the ferromagnet layer, and $\boldsymbol{j}$ is the current density.

We utilize a finite difference method based on the midpoint rule for the time discretization of Eq. (6) [17, 18]. After substituting Eq. (10) to Eq. (6), Eq. (6) can be rearranged to the following form:

$$
\frac{d \boldsymbol{m}}{d t}=-\frac{1}{1+\alpha^{2}} \gamma \mu_{0} \boldsymbol{m} \times \tilde{\boldsymbol{H}},
$$

where

$$
\begin{aligned}
\tilde{\boldsymbol{H}} & =\boldsymbol{H}+\alpha \boldsymbol{m} \times \boldsymbol{H}, \\
\boldsymbol{H} & =\boldsymbol{H}_{\mathrm{eff}}+\frac{\hbar}{2 e \mu_{0}} \frac{\theta_{\mathrm{sh}}}{t_{f} M_{s}}[\boldsymbol{m} \times(\hat{z} \times \boldsymbol{j})] .
\end{aligned}
$$

For the time discretization of Eq. (11), we introduce the midpoint rule approximations as follows:

$$
\begin{aligned}
\left(\frac{d \boldsymbol{m}}{d t}\right)^{\left(n+\frac{1}{2}\right)} & =\frac{\boldsymbol{m}^{(n+1)}-\boldsymbol{m}^{(n)}}{\Delta t}+O\left(\Delta t^{2}\right), \\
\boldsymbol{m}^{\left(n+\frac{1}{2}\right)} & =\frac{\boldsymbol{m}^{(n+1)}+\boldsymbol{m}^{(n)}}{2}+O\left(\Delta t^{2}\right),
\end{aligned}
$$


where the superscript $(n)$ denotes the time step $t_{n}$ and $\Delta t$ is the time interval $t_{n+1}-t_{n}$. We also need to approximate $\tilde{\boldsymbol{H}}^{\left(n+\frac{1}{2}\right)}$ in order to derive the formula of the time discretization. $\tilde{\boldsymbol{H}}^{\left(n+\frac{1}{2}\right)}$ is calculated by the extrapolation method as follows:

$$
\tilde{\boldsymbol{H}}^{\left(n+\frac{1}{2}\right)}=\frac{3}{2} \tilde{\boldsymbol{H}}^{(n)}-\frac{1}{2} \tilde{\boldsymbol{H}}^{(n-1)}+O\left(\Delta t^{2}\right) .
$$

By substituting Eqs. (13), (14), and (15) to Eq. (11) and neglecting $O\left(\Delta t^{2}\right)$, a time-discretized LLG equation is given by

$$
\begin{aligned}
\boldsymbol{m}^{(n+1)}-\boldsymbol{m}^{(n)}=-\frac{\gamma \mu_{0} \Delta t}{2\left(1+\alpha^{2}\right)} & \left(\boldsymbol{m}^{(n+1)}+\boldsymbol{m}^{(n)}\right) \\
& \times\left(\frac{3}{2} \tilde{\boldsymbol{H}}^{(n)}-\frac{1}{2} \tilde{\boldsymbol{H}}^{(n-1)}\right),
\end{aligned}
$$

which is a linear vector equation for $\boldsymbol{m}^{(n+1)}$. Therefore, it is easy to calculate the value of $\boldsymbol{m}^{(n+1)}$ using the values of $\boldsymbol{m}^{(n)}, \tilde{\boldsymbol{H}}^{(n)}$, and $\tilde{\boldsymbol{H}}^{(n-1)}$. Moreover, Eq. (16) has the desirable property that the magnitude of magnetization unit vector is conserved at any time steps. This is because the following equation can be obtained by calculating the inner product of both sides of Eq. (16) with the vector $\boldsymbol{m}^{(n+1)}+\boldsymbol{m}^{(n)}$ :

$$
\left|\boldsymbol{m}^{(n+1)}\right|^{2}=\left|\boldsymbol{m}^{(n)}\right|^{2} .
$$

After preparing the space and time discretization schemes mentioned above, we can perform the micromagnetic simulation by solving Eq. (16).

Meanwhile, we can also solve the LLG equation analytically under the special condition although it is impossible to solve the LLG equation analytically under the general condition. If it is assumed that the motion of magnetization structure is only translation and its configuration does not change, the dynamics of the magnetization is described by the Thiele equation which can be derived from the LLG equation [19]. From Eq. (6), we obtain the Thiele equation which describe steady state motion of a skyrmion written by

$$
\boldsymbol{G} \times \boldsymbol{v}-4 \pi \alpha \mathcal{D} \cdot \boldsymbol{v}+4 \pi \mathcal{B} \cdot \boldsymbol{j}=0
$$

where $\boldsymbol{G}=(0,0,-4 \pi Q)$ is the gyromagnetic coupling vector, $\boldsymbol{v}$ is the magnetic skyrmion velocity, $\mathcal{D}=\left(\begin{array}{ll}D & 0 \\ 0 & D\end{array}\right)$ is the dissipative force tensor, $\mathcal{B}=\left(\begin{array}{cc}B & 0 \\ 0 & B\end{array}\right)$ quantifies the efficiency of the spin Hall torque, and the components of these tensor $D$ and $B$ are determined by the configuration of the magnetic skyrmion. When the current density is spatially homogeneous and has only $x$-component $\boldsymbol{j}=\left(j_{x}, 0\right)$, the magnetic skyrmion velocity is given by

$$
v_{x}=\frac{\alpha D}{Q^{2}+\alpha^{2} D^{2}} B j_{x}, \quad v_{y}=-\frac{Q}{Q^{2}+\alpha^{2} D^{2}} B j_{x} .
$$

Since the skyrmion charge of a skyrmion is $Q=+1$ or $Q=-1$, the skyrmion has the perpendicular velocity component to the current flow. Moreover, the analytical ratio of in-plane velocity components is given by

$$
\frac{v_{y}}{v_{x}}=\frac{-Q}{\alpha D} .
$$

In order to estimate this value, we need to calculate the value of $D$. Generally, the components of dissipative force tensor $\mathcal{D}$ are given by $[11,19]$

$$
D_{i j}=\frac{1}{4 \pi} \int \frac{\partial \boldsymbol{m}}{\partial x_{i}} \cdot \frac{\partial \boldsymbol{m}}{\partial x_{j}} d x d y
$$

Using the magnetization of an isolated skyrmion described by Eq. (1), we obtain the components of $\mathcal{D}$ as follows:

$$
\begin{aligned}
& D_{x x}=D_{y y}=D, \\
& D_{x y}=D_{y x}=0,
\end{aligned}
$$

where

$$
D=\frac{1}{4} \int_{0}^{\infty}\left[\left(\frac{d \theta}{d r}\right)^{2}+\frac{n^{2} \sin ^{2} \theta}{r^{2}}\right] r d r
$$

and $n$ is vorticity. By substituting the solution of Eq. (3) into Eq. (24), we can obtain the value of $D$ of 1.204 .

\section{Results and discussion}

In micromagnetic simulation, the heavy metal and the ferromagnetic material are assumed to be tantalum and FeCoB. We use the following material parameters: $M_{s}=$ $600 \mathrm{kA} / \mathrm{m}, A=30 \mathrm{pJ} / \mathrm{m}, D_{\mathrm{DMI}}=4 \mathrm{~mJ} / \mathrm{m}^{2}, K=0.8 \mathrm{MJ} / \mathrm{m}^{3}$, $t_{f}=1 \mathrm{~nm}, \theta_{\mathrm{sh}}=-0.1$. First of all, we have to prepare a skyrmion structure for the initial condition before performing a calculation of skyrmion Hall effects. Then, a skyrmion structure can be obtained by the variational method. The red (blue) line in Fig. 2(a) shows the solution of Eq. (3) under the boundary condition $\theta(0)=0$ and $\theta(\infty)=\pi(\theta(0)=\pi$ and $\theta(\infty)=0)$. Moreover, the red and blue lines correspond to the skyrmions having the skyrmion charge of $Q=+1$ and $Q=-1$, respectively. In order to obtain the skyrmions size, we define a skyrmion radius as a point where the angle of $\theta(r)$ becomes $\pi / e$. Then, it is found that the radii of both the skyrmions are $11.3 \mathrm{~nm}$. Figures $2(b)$ and $2(c)$ show the skyrmion structures with skyrmion charge of $Q=+1$ and $Q=-1$, respectively. These skyrmion structures can be obtained from Eq. (1) by substituting the calculated $\theta(r)$. In both the figures, the directions and the colors of arrows represent the magnetization-direction and the magnitude of $z$-components of magnetization at each point, respectively. These skyrmions are called Néel-type skyrmions. This is because the magnetization-direction changes from up to down (from down to up) in the same way as Néel-type domain walls, which is clear from the cross sectional views of the skyrmions as shown in Figs. 2(d) and 2(e). 
Next, we utilize the above skyrmions for the initial states of the micromagnetic simulations. In the simulations, we apply the current density $j_{x}=1 \mathrm{TA} / \mathrm{m}^{2}$ to the heavy metal layer in the $x$-direction and the magnetic field $B_{z}=1 \mathrm{mT}$ in the $z$-direction. In the case of $\alpha=0.3$, the snap shots of time evolution of the skyrmion motions are shown in Figs. 3(a), 3(b), and 3(c) for the skyrmion with the skyrmion charge of $Q=+1$ (in Figs. $3(d), 3(e)$, and $3(f)$ for the skyrmion with the skyrmion charge of

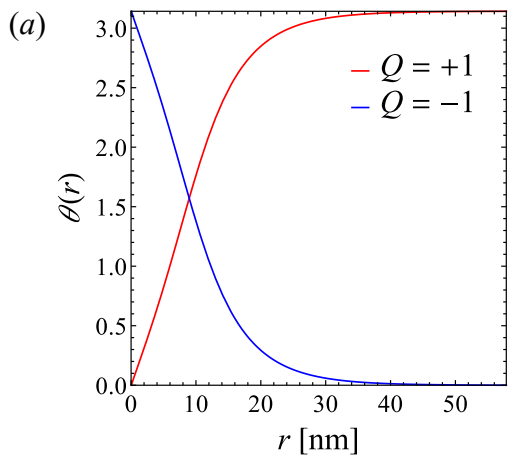

(b)

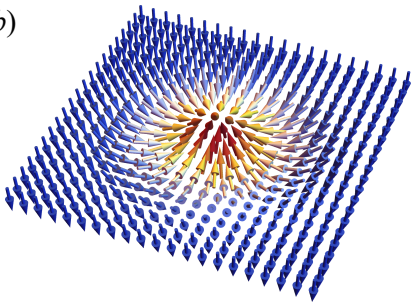

(c)

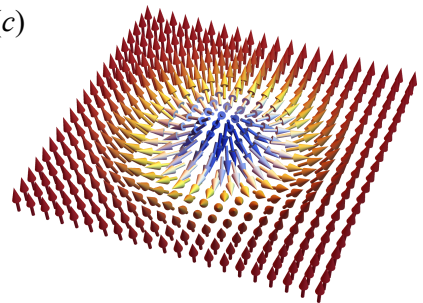

(d)

(e)

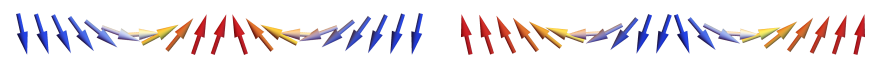

Figure 2: (a) The radial dependence of the $\theta(r)$ obtained using the variational method. The red and blue lines represent $\theta(r)$ of a $Q=+1$ skyrmion and a $Q=-1$ skyrmion, respectively. The magnetization structures of $(b)$ the $Q=+1$ skyrmion and $(c)$ the $Q=-1$ skyrmion obtained using the above $\theta(r)$, respectively. The cross sectional views of $(d) Q=+1$ skyrmion and $(e) Q=-1$ skyrmion. In figures $(b),(c),(d)$, and $(e)$, the directions and the colors of arrows represent the magnetization-direction and the magnitude of $z$ component of magnetization at each point, respectively.
$Q=-1)$. As shown in the figures, the skyrmions having the skyrmion charge of $Q=+1$ and $Q=-1$ deflect to the left and the right direction against the current flow, respectively. These results could be also obtained using the Eq. (19) which is derived from the Thiele equation. However, there exists the difference quantitatively between results obtained by the LLG equation and analytical solution by the Thiele equation. We can confirm the above discrepancy by comparing the ratios of in-plane skyrmion velocity components.

When estimating the ratio of in-plane skyrmion velocity components using the equation (20), we need to calculate the value of $D$. In order to calculate the value of $D$, we use the initial magnetization distribution of the skyrmion obtained by the variational principle. As a result, using Eq. (24), we obtain the value of $D$ of 1.204 . Then, this value of $D$ is assumed to remain constant since the equation (20) is derived from the Thiele equation analytically. We regard this an analytical ratio of in-plane skyrmion velocity components. On the other hand, in order to calculate the numerical value of the ratio, we utilize the average skyrmion velocities in the micromagnetic simulation. The analytical and numerical values are represented by the orange solid line and the blue solid circles in Fig. 4. As shown in the figure, the analytical ratio of in-plane skyrmion velocity components obtained using the Thiele equation deviates from the numerical one obtained using micromagnetic simulation with decreasing the value of the Gilbert damping constant $\alpha$. Since the Thiele equation is derived from the LLG equation assuming that the skyrmion structure does not change and behaves like a rigid body, the above result suggests that the system does not satisfy the assumption and that the Thiele equation is invalid when the value of $\alpha$ is relatively small. This is because the structures of skyrmions are distorted with decreasing the value of $\alpha$. As a result, the value of $D$ cannot remain constant in the equation (20) and the deviation from numerical results becomes large. Namely, under the (a)

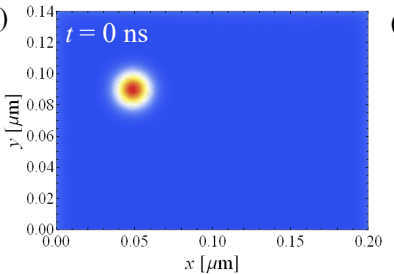

(d)

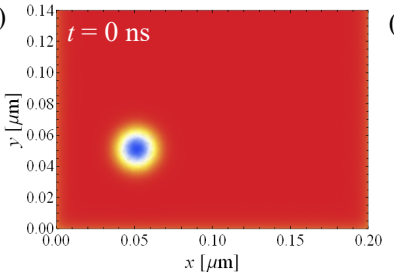

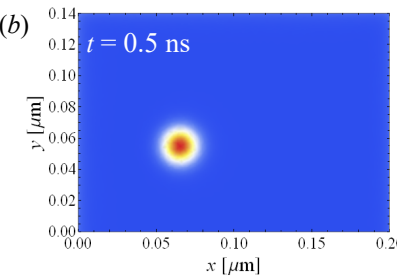

(e)

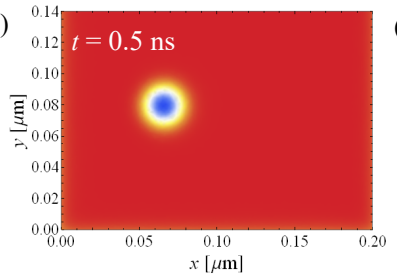

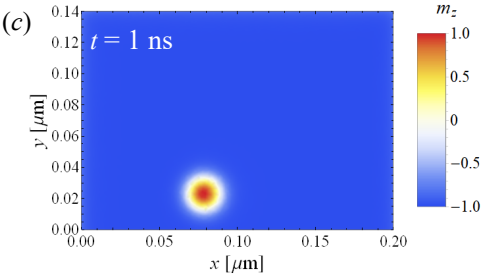

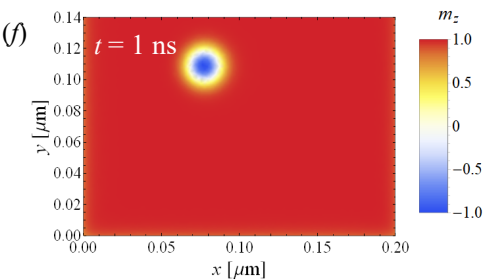

Figure 3: The time evolution of skyrmion motion $(a),(b)$, and (c) with the skyrmion charge of $Q=+1$ and $(d),(e)$, and $(f)$ with the skyrmion charge of $Q=-1$ in the case of $\alpha=0.3$. 


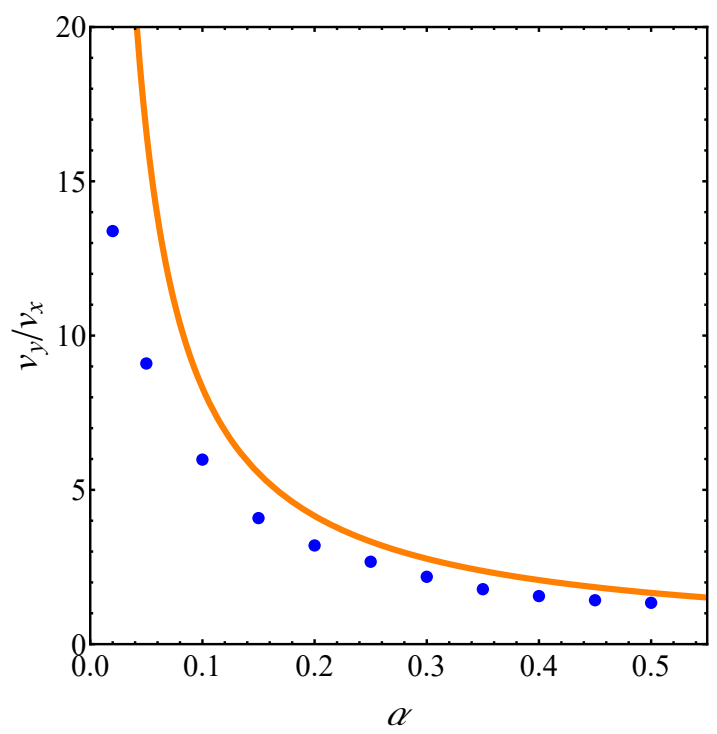

Figure 4: The dependence of the ratios of in-plane velocity components on the damping constant $\alpha$. The blue solid circles and the orange solid line represent the numerical results and analytical value obtained from Eq. (20), respectively.

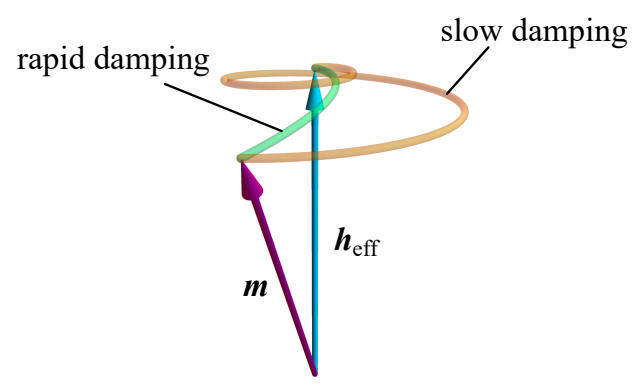

Figure 5: The illustration of the magnetization damping. Here, the red and blue solid arrows are the magnetization unit vector $\boldsymbol{m}$ and the effective magnetic field unit vector $\boldsymbol{h}_{\text {eff }}$, respectively. The green and the orange solid curves represent the trajectories of the magnetization for the large and small $\alpha$, respectively.

condition of smaller $\alpha$, the direction of the magnetization inclines more slowly to the direction of the effective magnetic field as shown in Fig. 5. This fact means that the structures of skyrmions are shaken for a long period to distort largely. Therefore, the dissipative force tensor $\mathcal{D}$ is considered to change from the initial state. In fact, Figure 6 shows time average values of the components of $\mathcal{D}$ which is calculated using Eq. (21) at the each value of $\alpha$. As shown in Fig. 6, the values of off-diagonal components of $\mathcal{D}$ become to have some values although they are equal to zero in the initial state as shown in Eq. (23). Therefore, we are sure that the skyrmion distortion is related to the off-diagonal part of the components of the dissipative force tensor. However, it is difficult to extract simple relation between the skyrmion distortion and the dissipative force tensor. We manage to extract simple relation between the skyrmion distortion and the dissipative force tensor. Then, we focus on the speed of the time change of the dissipative force tensor. In order to estimate the speed (a)

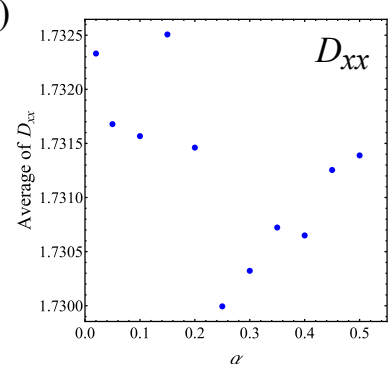

(c)

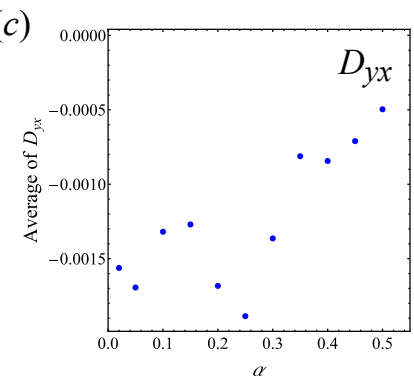

(b)

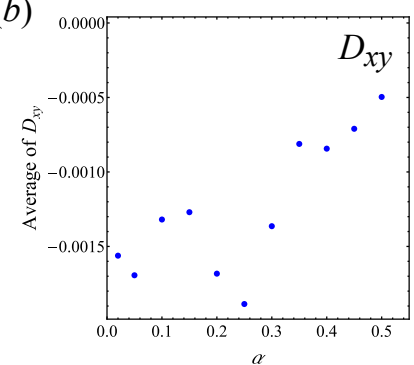

(d)

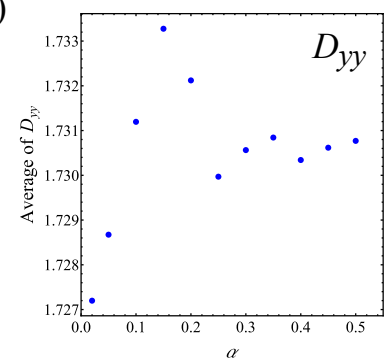

Figure 6: The time average values of the components of the dissipative force tensor $\mathcal{D}$ which is calculated using Eq. (21) for the different values of the damping constant $\alpha$.

(a)

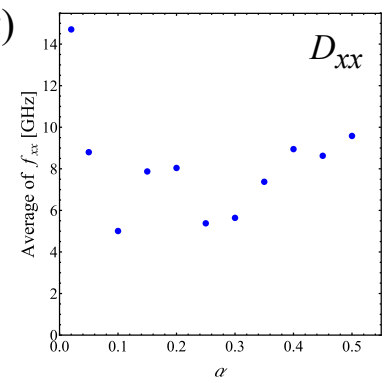

(c)

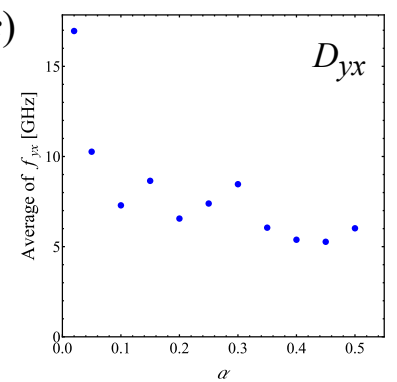

(b)

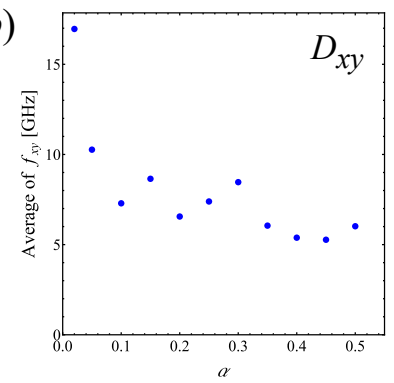

(d)

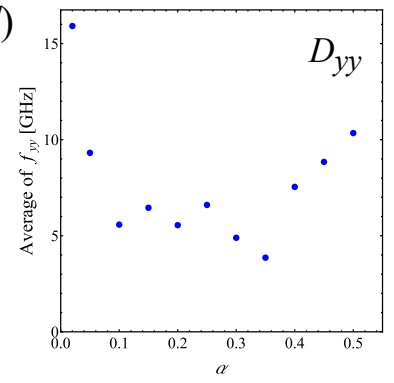

Figure 7: The average frequencies of the time change of the components of the dissipative force tensor $\mathcal{D}$ for the different values of the damping constant $\alpha$.

of the time change of the dissipative force tensor, we need to calculate frequencies of the time change of $\mathcal{D}$ using the DFT (discrete Fourier transform) method. Figure 7 shows the average frequencies for the different values of $\alpha$. As shown in Fig. 7, there exist a tendency that the frequency of the off-diagonal components of $\mathcal{D}$ becomes higher with the value of $\alpha$ decreasing. This means that the shape of the skyrmion vibrates drastically with the value of $\alpha$ decreasing. This fact is consistent with our above intuitive consideration. Therefore, we consider that the speed of the 


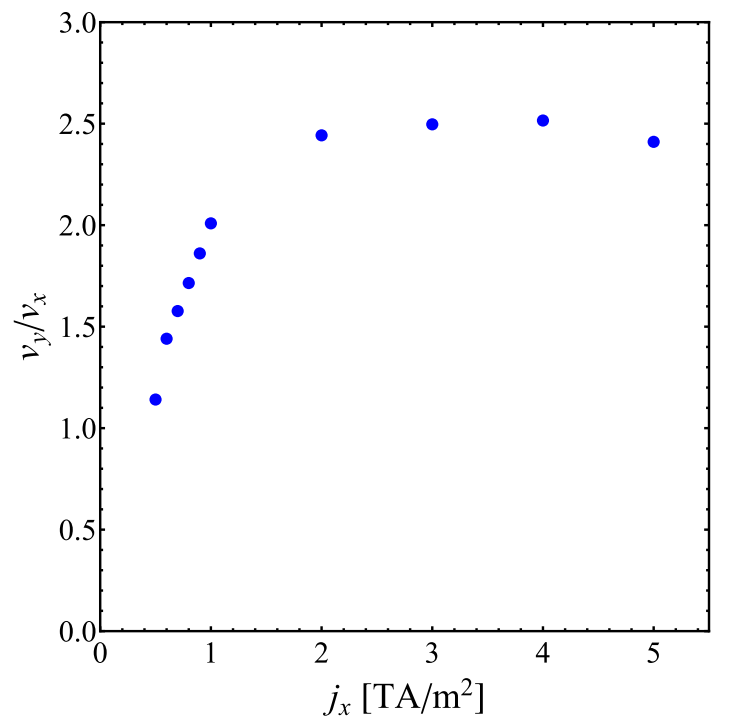

Figure 8: The dependence of the ratios of in-plane skyrmion velocity components on the current density $j_{x}$, which is obtained by utilizing the micromagnetic simulation.

time change of the skyrmion distortion is one of the reason for the difference between analytical value and numerical value.

Although, so far, we have discussed the dependence of the ratio of the skyrmion velocity components on the Gilbert damping constant, the ratio of the skyrmion velocity components also depends on the current density as shown in Fig. 8. Figure 8 shows the dependence of the ratios of in-plane skyrmion velocity components on the current density in the case of $\alpha$ of 0.3 , which is obtained by utilizing the micromagnetic simulation. This result is consistent with the result of Ref. [5].

\section{Conclusion}

When skyrmions are moved by the spin Hall torque, the motions of skyrmions are not parallel to the injected current flow and skyrmions deflect to the left or the right corresponding to the skyrmion charge. Namely they show so-called skyrmion Hall effect. This result is obtained using not only the micromagnetic simulation but also the Thiele equation. However, there exists clear difference between the ratio of the in-plane skyrmion velocity using the micromagnetic simulation and the one using the Thiele equation when the Gilbert damping constant is relatively small. This fact shows that the Thiele equation is invalid for the systems with the relatively small Gilbert damping constant. This is very important since the Gilbert damping constant of metal materials are generally very small. Therefore, when applying skyrmions to spintronics devices, it is mandatory to investigate the skyrmion dynamics accurately by utilizing the micromagnetic simulation.

\section{Acknowledgements}

This work is partially supported by a Grant-in-Aid for Scientific Research (Grant No. 16K04872) from JSPS, Center for Spintronics Research Network (CSRN) Tohoku University, and Dynamic Alliance for Open Innovation Bridging Human, Environment and Materials.

\section{References}

[1] N. Nagaosa, Y. Tokura, Topological properties and dynamics of magnetic skyrmions, Nature Nanotechnology 8 (2013) 899, review Article.

[2] A. Fert, V. Cros, J. Sampaio, Skyrmions on the track, Nature Nanotechnology 8 (2013) 152. doi:10.1038/nnano.2013.29.

[3] S. Maekawa, H. Adachi, K.-i. Uchida, J. Ieda, E. Saitoh, Spin current: Experimental and theoretical aspects, Journal of the Physical Society of Japan 82 (10) (2013) 102002. doi:10.7566/JPSJ.82.102002.

[4] A. Hoffmann, Spin hall effects in metals, IEEE Transactions on Magnetics $49 \quad$ (10) (2013) 5172-5193. doi:10.1109/TMAG.2013.2262947.

[5] W. Jiang, X. Zhang, G. Yu, W. Zhang, X. Wang, M. Benjamin Jungfleisch, J. E. Pearson, X. Cheng, O. Heinonen, K. L. Wang, Y. Zhou, A. Hoffmann, S. G. E. te Velthuis, Direct observation of the skyrmion hall effect, Nature Physics 13 (2016) 162, article. doi:10.1038/nphys 3883 .

[6] A. Bocdanov, A. Hubert, The properties of isolated magnetic vortices, physica status solidi (b) 186 (2) (1994) 527-543. doi:10.1002/pssb.2221860223.

[7] A. N. Bogdanov, U. K. Rößler, Chiral symmetry breaking in magnetic thin films and multilayers, Phys. Rev. Lett. 87 (2001) 037203. doi:10.1103/PhysRevLett.87.037203.

[8] A. Bogdanov, A. Hubert, The stability of vortex-like structures in uniaxial ferromagnets, Journal of Magnetism and Magnetic Materials 195 (1) (1999) $182 \quad$ - 192. doi:https://doi.org/10.1016/S0304-8853(98)01038-5.

[9] A. Bogdanov, A. Hubert, Thermodynamically stable magnetic vortex states in magnetic crystals, Journal of Magnetism and Magnetic Materials 138 (3) (1994) 255 - 269. doi:https://doi.org/10.1016/0304-8853(94)90046-9.

[10] T. L. Gilbert, A phenomenological theory of damping in ferromagnetic materials, IEEE Transactions on Magnetics 40 (6) (2004) 3443-3449. doi:10.1109/TMAG.2004.836740.

[11] W. Jiang, G. Chen, K. Liu, J. Zang, S. G. te Velthuis, A. Hoffmann, Skyrmions in magnetic multilayers, Physics Reports 704 (2017) $1-49$, skyrmions in Magnetic Multilayers. doi:https://doi.org/10.1016/j.physrep.2017.08.001.

[12] S. Rohart, A. Thiaville, Skyrmion confinement in ultrathin film nanostructures in the presence of dzyaloshinskiimoriya interaction, Phys. Rev. B $88 \quad$ (2013) 184422. doi:10.1103/PhysRevB.88.184422.

[13] R. Tomasello, E. Martinez, R. Zivieri, L. Torres, M. Carpentieri, G. Finocchio, A strategy for the design of skyrmion racetrack memories, Scientific Reports 4 (2014) 6784, article. doi:10.1038/srep06784.

[14] W. Jiang, P. Upadhyaya, W. Zhang, G. Yu, M. B. Jungfleisch, F. Y. Fradin, J. E. Pearson, Y. Tserkovnyak, K. L. Wang, O. Heinonen, S. G. E. te Velthuis, A. Hoffmann, Blowing magnetic skyrmion bubbles, Science 349 (6245) (2015) 283-286. doi:10.1126/science.aaa1442.

[15] S. Emori, U. Bauer, S.-M. Ahn, E. Martinez, G. S. D. Beach, Current-driven dynamics of chiral ferromagnetic domain walls, Nature Materials 12 (2013) 611.

[16] O. Heinonen, W. Jiang, H. Somaily, S. G. E. te Velthuis, A. Hoffmann, Generation of magnetic skyrmion bubbles by inhomogeneous spin hall currents, Phys. Rev. B 93 (2016) 094407. doi:10.1103/PhysRevB.93.094407. 
[17] C. Serpico, I. D. Mayergoyz, G. Bertotti, Numerical technique for integration of the landau-lifshitz equation, Journal of Applied Physics 89 (11) (2001) 6991-6993. doi:10.1063/1.1358818.

[18] O. Bottauscio, M. Chiampi, A. Manzin, A finite element procedure for dynamic micromagnetic computations, IEEE Transactions on Magnetics 44 (11) (2008) 3149-3152. doi:10.1109/TMAG.2008.2001666.

[19] A. A. Thiele, Steady-state motion of magnetic domains, Phys. Rev. Lett. 30 (1973) 230-233. doi:10.1103/PhysRevLett.30.230. 